\title{
The Application of Building Information Modeling in Interior Design
}

\author{
Hongxing $\mathrm{Yi}^{1}{ }^{1, \mathrm{a}}$ and Wenjun Wang ${ }^{1, \mathrm{~b}}$ \\ ${ }^{1}$ City College of WUST, Wuhan, China \\ aYihongxing2016hy@163.com, bwangwenjun2016hy@163.com
}

Keywords: Building information; Interior design; Information technology; Digital

\begin{abstract}
The advent of the computer as a symbol of the information age has brought great changes to all aspects of modern society. Interior Design as a new industry, but also inevitably be affected. Interior Design With the development of the information age, showed the following trends: digital, exchange of information and convenient design tools, intelligent living space. Designers not only the information age to the computer as a medium of modern design, is more important is to have quickly and accurately grasp, control, and the ability to use a variety of information. This article describes the specific meaning of its technical idea of building information modeling, and discusses the application of revolutionary changes in its interior design brings, and two examples further illustrate the advantages of building information modeling technology in interior design applications.
\end{abstract}

\section{Interior Design in Information Age}

Information Age. Advances in technology bring us into a new era of information. Around the mid-20th century, with the emergence of the first computer, it heralded the advent of the information age. With the decline of the agricultural age and the industrial age, human society is the transition to the information age, entered the Third Wave civilization, its social form is developed from an industrial society to an information society. The biggest difference between the two previous wave of the Information Society and the Third Wave of agricultural society and the industrial society is no longer based on physical and mechanical energy, but in a smart based.

Interior Design. Modern interior design as an emerging discipline, its history is long. Interior design is accompanied by the generation of construction and the progressive development, perhaps the first hominids to take the initiative to decorate their room from the start. Enjoy beautiful Broadly speaking, the interior design is to meet people's spiritual and material life requirements, and thus the production of human environment, living environment, work environment, physical and spiritual transformation, to achieve the necessary conditions for the use of functional and visual environment . Interior design style formation, different ideas and regional characteristics of the times, through creative ideas and expressions, and gradually developed into a representative form of interior design. Interior designer information age, not be confined to a particular style, but should be bold trend of the times, we feel the technology brings convenience and comfort. Interior Design Information Age, not only advances in design tools, information exchange and other aspects of the more obvious trend are the intelligent living space. This is bound to become a major trend in the future development of interior design.

Building Information Modeling. Building information modeling technology should include the meaning and application modeling two aspects: from a modeling point of view, the building information model is based on three-dimensional digital technology, integrated construction projects in various engineering data model-related information, is detailed information related to the project of digital expression. It contains information about the geometry of three-dimensional model building, the project also includes the physical and functional properties of building components and their associated lifecycle information. From an application standpoint, the building information model emphasizes the complete digitization of information requirements, we can always add to the model in a variety of engineering information in the life of the project, to meet the various needs of the project.

BIM-based design software technology contains the following three main technical ideas: first, 
in three-dimensional space to establish a single, digital building information model, all the buildings are unified information database is stored in the form of the model, in order to be updated and shared. Thus, all design drawings by the same digital building model generated charts are interrelated, can be achieved between the various entities associated with the digital component display, intelligent interaction. Any changes to the design data are immediately reflected in the other parts of the association. Third, support for data representation and transmission of information in many ways. BIM software supports both two-dimensional expression of the traditional way and chart flat, vertical, cross-sectional view, also support perspective view three-dimensional perspective and other ways to express even animated display, but also support XML to facilitate network transmission.

\section{Development Trend of Interior Design in Information Age}

Digital Design Tools. Information age, the computer has been widely applied to various fields of production and life. Interior design is also closely follow the trend of the times, so that interior designers to do the design faster and easier. Before the popularization of computers, thanks to the tedious hand-painted to complete construction drawings, laborious trouble, it is not easy to modify. Participation of the computer, and did not completely replace manual drafting, this is because the hand-painted randomness is more conducive to the performance of the initial program. However, the use of the computer does occupy the mainstream of modern interior design market. With the popularity of personal computers, a large number of interior design software also emerged. For example, the domestic market is now more commonly used several software, two-dimensional drawing software Auto CAD, 3D modeling software 3ds Max, rendering plug Vary. Use the software for interior designers to provide more forms, improving efficiency. Understandable, digital design tools to promote the development of the interior design industry. Computer Performance Chart can be a more realistic reflection of the final results. Whether it is from and other space, materials, or colors, realistic simulation is the information age led advocated.

Convenient Information Exchange. Information age, is an information exchange, resource sharing era. Interior designers can choose according to their own designs suitable material, and timely communication with material suppliers to discuss the operational program. Interior designers can also be timely and effective communication with customers through the network platform to allow customers to participate in the design to create with the design features, letting customer satisfaction designs. The popularity of computer networks, information superhighway construction, has brought great convenience to our lives today. We can stay at home, you can learn all kinds of information, because the network provides us with a communication platform. Working as a designer in the office and design company, you can choose the material, the model in another way, through the decorative material commercial sites, contact the manufacturer directly to obtain the latest information. Decorative materials manufacturer catalog prices also released outward through the network, DVD and other new carrier.

Intelligent Living Space. 21 century, the interior design has entered the information age. Intelligent life will lead the future direction of interior design. Smart home can be defined as an object or a system. The use of advanced computer, network communications, automatic control technology, family life and various application subsystems together organically, through integrated management, to make family life more comfortable, safe, effective and energy-saving. Compared with ordinary home, smart home features not only traditional living functions, but also provide comfort and safety, energy-efficient, highly humane living space; the number of original equipment passive static home into a tool with the "wisdom", comprehensive information exchange capabilities to help keep the family and external exchange of information flow, optimize people's way of life, helping people to organize time effectively, enhance the security of family life and family to save energy costs. 


\section{The Challenge of Traditional Interior Design}

Traditional computer-aided design interior mainly uses Auto CAD and other design software for two-dimensional flat, vertical, sectional view of the design, production in $3 \mathrm{ds}$ Max and other three-dimensional software to effect the performance of FIG. This mode of operation is similar to the traditional method of hand-drawing step, just use the computer as a medium, compared with manual drawing to efficient, accurate, not freed from the onerous task of drawing that fundamentally designers. Traditional design software not possible correlation between flat, vertical, sectional view and between the three-dimensional models, made some changes in the drawings, other drawings to give the corresponding modified manually, even redrawn, often have a variety of drawing expression of mutual inconsistent phenomenon. In order to refine the details of the final design and effect, designer finishes flat, vertical, sectional view after design, 3D model using 3ds Max software can render photorealistic renderings. Since using 3ds Max software modeling complex operations, designers difficult to master, with about 3ds Max modeling studies emerged. But there is no such three-dimensional model of integration-related information, the purpose is merely to obtain a three-dimensional browsing performance animation or effect map, its value cannot be brought into the construction design and project management phases later. The above problem is a common problem currently facing the computer aided interior design, is a serious challenge to further improve the level of design and design efficiency face. These problems can only be solved through the application of new computer technology, building information modeling technology to provide a reliable solution.

\section{The Advantages of Building Information Modeling Technology in Interior Design Application}

The BIM technology in interior design will bring revolutionary changes, mainly in the following several aspects.

Information Modeling. BIM design software is no longer available only draw points, lines, circles and other geometric elements of simple drawing tools, but in the design process directly placed walls, doors, windows, beams, columns and other member entities, established by the member composition the information model. Software with a powerful component library, various components, furniture, kitchen equipment, sanitary ware are stored in a library, you can call at any time. After the model, it can automatically generate the required expression of a variety of interior design drawings.

Intelligent Interaction. Because of the various drawings are generated from the same model, all drawings, diagrams are interrelated, to avoid the phenomenon of inconsistency between different views. If you need to make changes to the design, no matter on which a view of the changes associated with the drawing, also followed on the chart associated with the change occurred, the association changed in real time. Changes associated with the intelligent interaction between members are also reflected in the presence of a relationship. For example, mounted on the ceiling in the model lamps, ceiling if we want to increase or decrease the height of the lamp will follow raise or lower; if the model remove the ceiling, lighting on the ceiling will also be deleted.

Visualization Analysis. Mentioned earlier, BIM design software is designed to establish the model results. As a variety of flat, vertical, sectional view, as well as three-dimensional effects, 3D animation and so can freely generate the model, which is designed for the visual analysis (spatial analysis, body mass analysis, effect analysis, details of the deal) provides a convenient. Designers can take advantage of a three-dimensional model of scrutiny on any design perspective, to determine material, color finishes, lighting layout, fixed facilities, pipelines and collision checking member, the identification of the exact location of the pipeline through the member, thereby leading to detailed analysis of the design, to ensure the quality of the design.

More Attention to Details. Interior design is very attention to detail, visual analysis to handle the details to create the conditions. Lighting, materials, finishes furniture and other details of the impact on the final results of the design. Whether in the conceptual design phase or deepen the design phase, BIM design software enables designers to vivid and easily show these details. 
Many Additional Features. Because BIM database contains detailed information on behalf of the building, thus creating conditions for further application of BIM, we added a lot of additional features for the design work. You can use it to automatically count quantities. Generate a variety of doors and tables, and a variety of materials from the comprehensive table model is very easy. To count a sepal wall area, the number of the volume, or decorative elements, price, manufacturer information is also very convenient. Designers can easily use it to carry out the project budget estimate for the control of decoration offer or tender offer provides accurate data to ensure that the range of the actual cost of the difference in rates can be controlled. Use it to generate purchase lists, etc. to ensure the accuracy of the purchase amount. In addition to interior space design functional design and the visual arts, but also including indoor sound, light, thermal environment design. Especially large public building interior space of sound, light, heat environment with high demands. Because the rich data contained in the BIM also includes a variety of physical data member material (heat resistance, sound insulation coefficient, etc.), which is directly derived information such as model to Octet, IES and other analysis software for sound, light, building physics thermal environment to create the conditions.

Information Sharing and Collaboration. In the building information model, data relating to all the basic elements of construction works are stored in a unified database, and information integration. This makes it possible to build the core BIM collaboration platform to make the interior design, electrical, plumbing and other various professional designers are able to expand the collaborative design on the same building model through the network, but also through collaborative work platform with the owner, construction suppliers, material suppliers and other coordination and communication and communicate information in a timely manner, information can be effectively managed and applications, designed to ensure coordinated and efficient, smoothly.

\section{Summary}

BIM-based technology developed 3D design software for the interior designer of the construction design, visual analysis of a design from concept to, renderings, and documentation unified environment, eliminating the inconsistencies between the design drawing phenomenon, designed to ensure coordination, efficient and smoothly to ensure the design quality and progress. Practice has proved that the design based on 3D BIM technology is a very effective means for the designer to analyze the three-dimensional spatial concept of a relation, and ultimately the expression of three-dimensional design to achieve the optimal design goals to achieve full use of design information. BIM design software application designer can change and enhance awareness of indoor space and build a form of understanding the significance of its design ideas and methods are gradually being recognized, also brought a new challenge for the interior design education, which Information technology is also being developed for design thinking to bring new shocks, new revolution.

\section{References}

[1] Yao Hui. Interior Design Teaching Digital Research. Prospective Technological .2016 (15)

[2] Peng Binbin fusion of interior design and natural greening. Modern decor (theory) .2016 (03)

[3] Rui Pengfei traditional modern interior design rendering. Modern decor (theory) .2016 (03)

[4] South Stream cultural connotation of consumer culture Perspective interior design values. Modern decor (theory) .2016 (05)

[5] Members Lina. Caisson in the interior design of functional studies .2016 of Art and Science (04)

[6] Lai Xiao Di interior design principles based on the concept of humanity. Packaging World .2016 (03) 